\title{
Fabrication and Evaluation of Ketorolac Loaded Cubosome for Ocular Drug Delivery
}

\author{
Zubair Ali, Pramod Kumar Sharma, Musarrat Husain Warsi* \\ Department of Pharmacy, School of Medical and Allied Sciences, Galgotias University, Plot No. 2, Sector 17-A, Yamuna Expressway, Greater Noida, \\ Gautam Budh Nagar, Uttar Pradesh, India.
}

\section{ARTICLE INFO}

\section{Article history:}

Received on: 14/05/2016

Revised on: 09/06/2016

Accepted on: 24/06/2016

Available online: 26/09/2016

Key words:

Ketorolac tromethamine, Glyceryl mono-oleate,

Poloxamer 407, Ocular Drug

Delivery.

\begin{abstract}
Ophthalmic formulations in terms of eye drops are more frequently used formulation for ocular disorders. But unfortunately this mode of drug instillation into the cul-de-sac of eye shows very poor ocular bioavailability (less than 5\%). A large number of carrier systems have been investigated to overcome this problem. In the present study a novel nano-carrier system (Ketorolac loaded cubosomes) is developed and evaluated for the safe and enhance ocular bioavailability. Cubosomes were developed and optimized by utilizing glyceryl monooleate, poloxamer 407 and initial drug concentration. Finally developed formulation was evaluated for various In vitro characteristics i.e. particles size, size distribution, shape and morphology, in-vitro release profile, corneal permeation, corneal retention, and ocular tolerance study. The optimized drug loaded cubosomal formulation showed mean particle size, polydispersity index, and entrapment efficiency $127.3 \pm 12.23 \mathrm{~nm}$, $0.205 \pm 0.011$, and $53.27 \pm 5.23 \%$, respectively. Transmission electron microscopic analysis revealed a cubic shape of developed formulation. Further, developed formulation exhibited biphasic release profile. Significant high transcorneal permeation (2.07 folds) and corneal retention ( 2.24 folds) of ketorolac was observed with cubosomal formulation correspond to Ketorolac solution $(\mathrm{p}<0.01)$. Further safety profile of optimized formulation was evaluated by histopathology of corneal membrane. The developed novel ocular carrier system (cubosomes) might be a promising platform as a vehicle for effective ocular drug delivery.
\end{abstract}

\section{INTRODUCTION}

Delivery of drugs to the ocular sight is a promising field for the formulation researchers. It is due to the complex anatomy and physiology of the eye. The most preferred route for the administration of drug is the topical instillation of formulation into cul-de-sac of eye to treat the ocular diseases like conjunctivitis, uveitis, endophthalmitis, glaucomatous conditions and post operative pain. in spite of of being a most preferred and convenient route of administration, still draw outs constraints owing to poor ocular bioavailability $(<5 \%)$.

\section{* Corresponding Author}

Dr. Musarrat H Warsi, Department of Pharmacy, School of Medical and Allied Sciences, Galgotias University, Plot No. 2, Sector 17-A, Yamuna Expressway, Greater Noida, Gautam Budh Nagar, Uttar Pradesh, India. Email:mhwarsi@gmail.com
The poor availability of drugs into the ocular chamber can be due to the high turnover rate of tears, lower corneal permeation and rapid naso-lachrymal drainage leads to fast decay in drug concentration in ocular environment (Warsi et al., 2014). For enhancing the ocular bioavailability, a number of formulations have been developed like gels or hydrogel,(Araujo et al., 2009) in situ gelling systems, (Badawi et al., 2008) ocuserts, (Bourlais et al., 1998) polymeric vehicles, (Anitha et al., 2011) . Hydrogels limit their value due to improper administration that is not reproducible. In situ gelling systems showed prolonged result as compared to free solution and suspension (Chien et al., 1992; Chopra et al., 2007). Gels or gelling systems may cause blurred vision and lachrymation. Ocusert revealed a sustained effect but it can cause crusting of eyelids, unintended elimination during sleep, and meddling with vision (Davies et al., 1992; De Campos et al., 2004). 
Nanoparticles materialized as effective approach to improve the ocular bioavailability of drugs into the ocular chamber. Polymeric nanoparticles are most explored system to enhance the ocular bioavailability, (Dodane et al., 1999; Felt et al., 1999; Guinedi et al., 2005) and the main focussed polymers for ocular delivery are poly(alkylcyanoacrylate), poly( $\mathcal{E}$-caprolactone), and PLA, PLGA Eudragits, polystyrene, and chitosan.(Gupta et al., 2010; Felt et al., 1999; Guinedi et al., 2005)

Cubosomes are dispersed colloidal particles of cubic phase liquid crystals. These have aroused significant importance as controlled-release drug delivery system. Cubosomes are generally prepared from a glycerol monooleate (GMO)-water mixture through high-pressure emulsification, using poloxamer 407 as a stabilizer (Malmsten et al., 2006). Cubosomes have various advantages as a drug delivery system, together with their capability to incorporate both hydrophilic and lipophilic drugs (Malmsten et al., 2006; Zhao et al., 2004), provide controlledrelease of drugs (Zhao et al., 2004). Cubosomes also imparts biocompatibility and bioadhesivity (Larsson et al., 2000; Garg et al., 2007).

The present work aimed to design and develop a novel carrier system based on cubosomes as an ocular drug delivery system of ketorolac that would provide ease of delivery, nonirritancy, high corneal permeation, sustained effect, and enhance bioavailability. The morphological properties of cubosomes were assessed by high resolution-TEM. The In vitro release profiles of ketorolac loaded cubosomes were studied by dialysis method, and the In vitro corneal penetration of the developed formulation was investigated and the probable harmful effect on ocular tissues (cornea) was assessed on the basis of histological examination.

\section{MATERIALS AND METHODS}

\section{Materials}

Ketorolac tromethamine was obtained as gift sample from Ranbaxy, Gurgaon, Haryana (India). Glyceryl monooleate (Brand name: Pecol) was a gift sample from Gattefose (France). Ploxamer 407 was purchased from Sigma Aldrich (Germany). Purified water obtained from Milli Q water purification system (Millipore, Billerica, MA, USA) was used for conducting all the experiments. All other chemicals and reagents used were of analytical grade.

\section{Preparation of Cubosomes}

Blank and Ketorolac loaded Cubosomes were prepared by fragmentation of glyceryl monooleate (GMO: $1 \mathrm{~mL}$ ) in Poloxamer $407(0.1 \mathrm{~g})$ and water $(1 \mathrm{~mL})$. Accurately weighed amounts of drug $(0.4 \%$ w/v) with the GMO and poloxamer 407 were melted at $60^{\circ} \mathrm{C}$. Milli-Q water was added gradually with continuous vortexing for achieving a homogenous state. This mixture was further stirred on magnetic stirrer at $300 \mathrm{rpm}$ and kept for $48 \mathrm{~h}$ to form cubic gel. Excess amount of Milli-Q water (20 $\mathrm{mL}$, q.s.) was gradually added under stirring and further sonicated in a bath sonicator for $3 \mathrm{~min}$. Finally, formulations were subjected to probe sonication for varying times using pulse mode $(9 \mathrm{~s}$ on and $18 \mathrm{~s}$ off) at $35 \%$ amplitude (using Vibra-Cell ${ }^{\mathrm{TM}} \mathrm{VC} 750 ; 400 \mathrm{~W}$, Sonics, USA). Developed formulation was lyophilized with $5 \%$ mannitol to get the final formulation and was evaluated for particle size and entrapment efficiency (Warsi et al., 2014).

\section{Characterization of cubosomes \\ Particle size and size distribution}

Mean particles size and size distribution were determined using dynamic light scattering method using Malvern Zeta Sizer and DTS software (Zetasizer, HAS 3000; Malvern Instruments, Malvern, UK). All the measurements were conducted in triplicate at a scattering angle of $90^{\circ}$ and $25^{\circ} \mathrm{C}$ temperature.

\section{Particle morphology}

Morphological analysis was carried out by transmission electron microscopy (TEM; TOPCON002B, Tokyo, Japan, accelerating voltage $200 \mathrm{kV}$ ). Image processing and study was done by Digital Micrograph and Soft Imaging Viewer software (Olympus, Singapore).

\section{Encapsulation efficiency}

The drug entrapment efficiency was determined by previously reported method (Abdullah et al., 2016) using ultracentrifuge filtration tubes $\left(\right.$ Amicon $^{\circledR}$ Ultra $3000 \mathrm{MWCO}$, Millipore, Germany). Diluted sample was placed in centrifuge tubes and centrifuged (REMI, Mumbai, India) for $15 \mathrm{~min}$ at 5000 rpm.

Filtrate was measured at absorption maxima $\left(\lambda_{\max }\right) 322$ $\mathrm{nm}$ by using UV-VIS, spectrophotometer (Shimadzu UV1800, Japan) and entrapment efficiency calculated by following equation;

$$
\begin{aligned}
& \% \text { Entrapment efficiency } \\
& =\frac{\text { Total amount of Ketorolac }- \text { unentrapped Ketorolac }}{\text { Total amount ofKetorolac }} \times 100
\end{aligned}
$$

\section{In vitro release analysis}

In vitro release profile of developed formulation was evaluated by dialysis bag method (Guinedi et al., 2005). Formulation (equivalent to $1 \mathrm{mg}$ of ketorolac) was suspended in 2 $\mathrm{mL}$ of simulated tears fluids (STF: KCl: 1.7893 ; $\mathrm{NaCl}$ : 6.3118; $\mathrm{NaHCO}_{3}: 2.1842 ; \mathrm{CaCl}_{2}: 0.0444 ; \mathrm{MgCl}_{2}: 0.0476 ; \mathrm{g} / \mathrm{L}$ ), placed into a dialysis bag (MW cut-off of $12 \mathrm{kDa}$ ), tied both the ends of bag and suspended it in beaker containing $20 \mathrm{~mL}$ of STF under stirring. Release study was carried out at $32 \pm 0.5{ }^{\circ} \mathrm{C}$ with stirring speed of $25 \mathrm{rpm}$. Samples $(2 \mathrm{~mL})$ were withdrawn at different predetermined time point and analyzed by using UV spectrophotometer at absorption maxima $\left(\lambda_{\max }\right) 322 \mathrm{~nm}$ (Shimadzu UV1800, Japan). Withdrawn volume of release medium was replaced with the fresh media to maintain sink condition. Similar practice was followed for marketed eye drops. All measurements were carried out in triplicate. 


\section{In vitro trans-corneal permeation study}

The goat cornea was excised from the freshly-excised goat eyes, which were obtained from a local slaughter house. The excised corneas were placed between the donor and receptor compartments of the Franz diffusion cell. In the receptor chamber STF ( $\mathrm{pH}$ 7.4) was used as the medium and the temperature was maintained at $32 \pm 2 \circ \mathrm{C}$. Samples $(0.4 \mathrm{~mL})$ were withdrawn from the sampling port of the receiver chamber at predetermined time intervals, i.e., 15, 30, 45, 60, 90, 120, and $180 \mathrm{~min}$, and replaced with an equal volume of STF. The withdrawn samples were further analysed by UV spectrophotometer (Warsi et al., 2014).

\section{In vitro corneal retention study}

Corneal (goat cornea) retention of ketorolac from developed formulation and marketed eye drops was performed comparatively as per the previous reported method (Abdullah et al., 2016). Initially, cornea was mounted between donor and receptor compartment of Franz diffusion cell and allowed to stabilize with prewarmed $(32 \pm 2 \circ \mathrm{C})$ simulated tear fluid for 15 min. Now, STF of donor chamber was replaced with $100 \mu \mathrm{L}$ of marketed eye drops and equivalent amount of NPs suspension in STF. After $2 \mathrm{~h}$, exposed cornea under study was taken out and rinsed properly with STF. Further this cornea was homogenized in an equivalent amount of STF followed by extraction in methanol. The obtained homogenate was further subjected to centrifugation at $10,000 \mathrm{rpm}$ for $10 \mathrm{~min}$. The resultant supernatant was filtered through membrane filter $(0.45 \mu)$ and quantitatively analyzed at absorption maxima $(\lambda \max ) 322 \mathrm{~nm}$ by UV spectrophotometer (Shimadzu UV1800, Japan).

\section{Ocular tolerance studies}

\section{Histopathology study}

Ex-vivo ocular tolerability was examined by accessing the histological cross-sections of the excised goat cornea under light microscopy (Jain et al., 2011). Any changes in the cornea due to the developed formulation (irritation potential) were further studied. The corneas were incubated in the formulation, positive $(1 \% \mathrm{w} / \mathrm{v} ; \mathrm{SLS})$ and negative control $(0.9 \% \mathrm{w} / \mathrm{v} ; \mathrm{NaCl})$ for a 1 hour and then washed with STF and immediately fixed with a $10 \%$ (v/v) formalin solution. The corneal tissue was treated with alcohol and putted in melted paraffin and solidified into a block form. Cross-sections were made, stained with haematoxylin and eosin, and examined microscopically for any modifications in the tissue structure.

\section{RESULTS AND DISCUSSION}

\section{Preparation of cubosomes}

Blank and Ketorolac loaded cubosomal nanoparticle dispersions were prepared by fragmentation of GMO in poloxamer and water. The dispersions emerged as uniform dense white mixtures with no visible signs of aggregate. It was observed that cubosomes of this composition have reasonable physicochemical properties with minimum particle size and maximum encapsulation efficiency.

\section{Characterization of cubosomes}

Particles size measurement of the developed formulation was determined by dynamic light scattering particle size analyser and the mean particle size was found to127.3 $\pm 12.23 \mathrm{~nm}$ with poly dispersity index $0.205 \pm 0.011$ (Fig. 1)

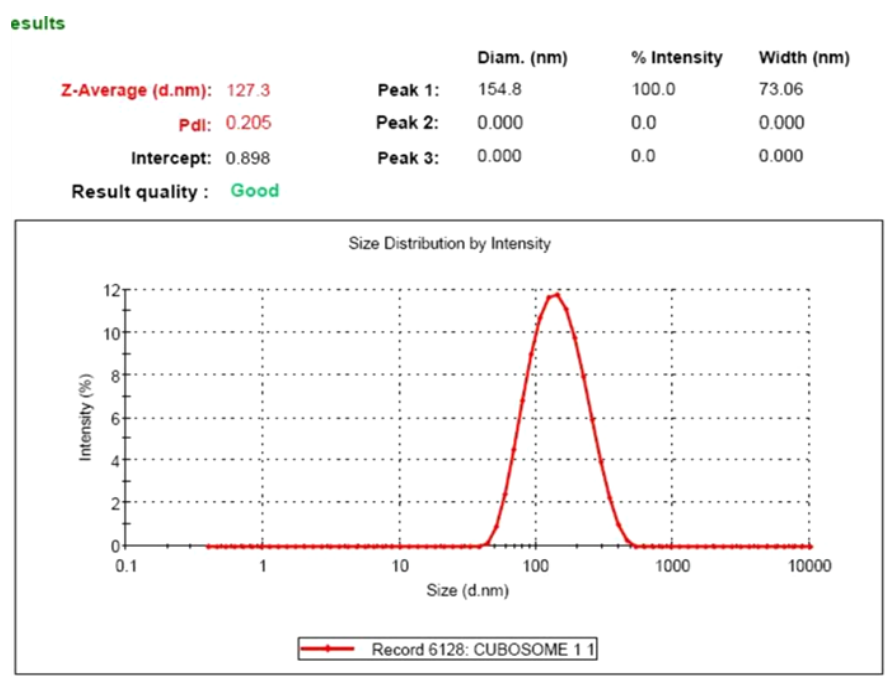

Fig. 1: Particle size and PDI report of optimized Ketorolac loaded cubosome.

The morphological evaluation of Ketorolac loaded cubosomal nanoparticles was achieved using TEM. The Image of TEM (Fig. 2) revealed that the drug-loaded cubosomal nanoparticles were polyangular shapes without aggregation. Evaluation of the particle size from the TEM images was in good agreement with the values obtained by laser light scattering method

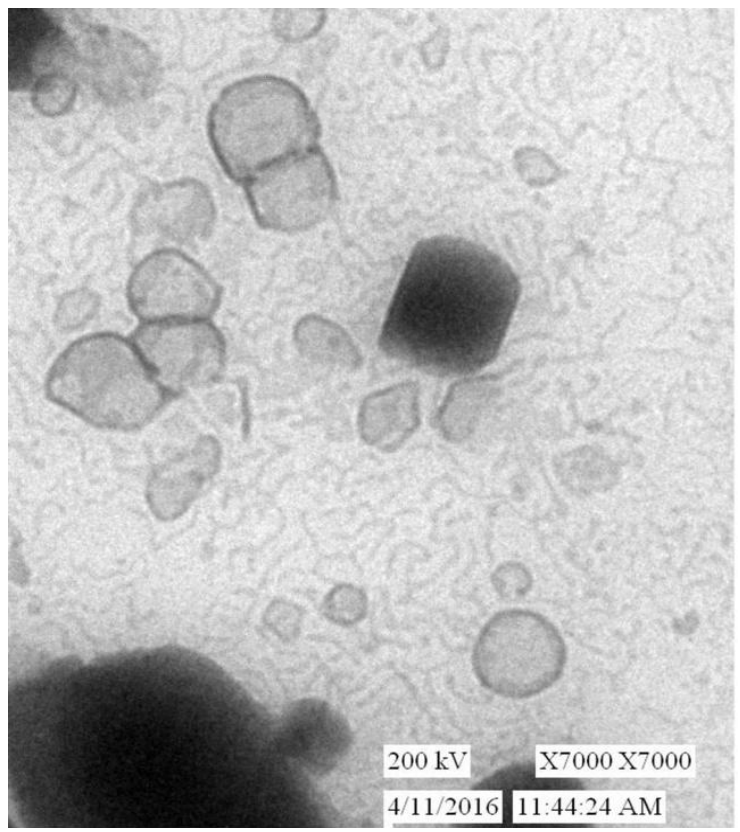

Fig. 2. HR-TEM image of Ketorolac loaded cubosome. 
EE of Ketorolac in cubosomes was determined after parting of the free Ketorolac from drug loaded cubosomal nanoparticles by ultra-centrifugation. The EE was found to be $53.27 \pm 5.23 \%$. The obtained EE of Ketorolac may be attributed to the nature of the drug molecule, which does not bracket together with the lipid bilayer (Tsukada et al., 1984) and was anticipated to be entrapped within the aqueous channels of cubosomes (Boyd et al., 2003). Mean particle sizes of blank and drug loaded cubosomes were $109.8 \mathrm{~nm}$ and $127.3 \mathrm{~nm}$, respectively. The polydispersity indices of blank and drug loaded cubosomal dispersions were 0.125 and 0.205 , respectively. The formation of bilayers monoolein/poloxamer leads to cubosomal arrangements with high poloxamer 407 which sterically stabilize the particles into the cubic state along with the formation of smaller particles. (Gustafsson et al., 1996; Worle et al., 2007).

\section{In vitro drug release study}

In vitro drug release profiles are reported for Ketorolac loaded cubosomes compared to Ketorolac solution as illustrated in Fig. 3. A hasty and complete release was observed from Ketorolac solution after $2 \mathrm{~h}$. The release profile of Ketorolac loaded cubosomes was found to be biphasic, with initial burst release of around $41.48 \pm 1.88 \%$ of drug during the first $1.5 \mathrm{~h}$, followed by a moderately slow release of the remaining drug for a period of $20 \mathrm{~h}$. The higher initial burst release is mainly due to the adsorbed drug or weakly bound drug to the relatively larger surface of cubosomes. The burst release of hydrophilic and hydrophobic drugs from cubosomes was previously reported (Magenheim et al., 1993).

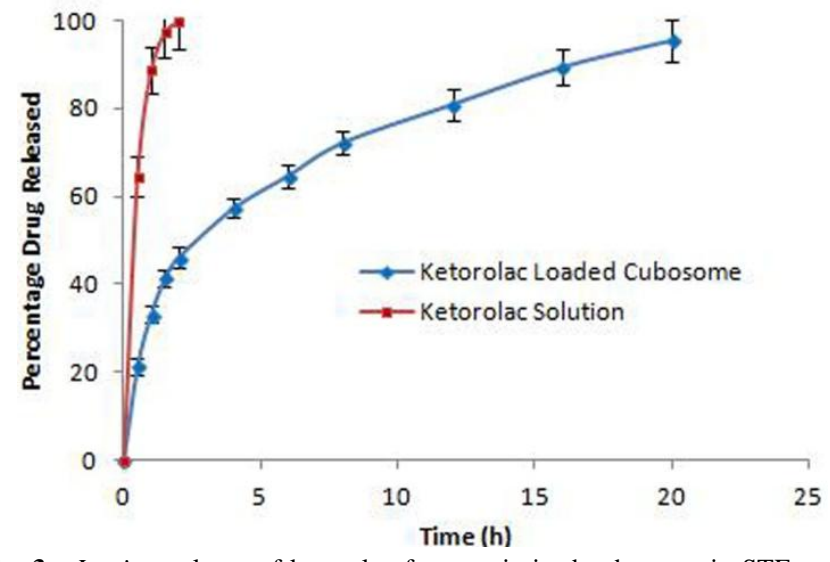

Fig. 3: In vitro release of ketorolac from optimized cubosome in STF at $\mathrm{pH}$ 7.2 ,

\section{In vitro corneal permeation evaluation}

The transcorneal permeation profiles of Ketorolac are shown in Fig. 4. Ketorolac crosses the cornea at a significantly higher rate with the application of Cubosomal formulation as compared with the Ketorolac solution ( $p<0.01$ ). At 4 h, $11.5 \pm$ $0.84 \%$ and $5.6 \pm 0.73 \%$ of Ketorolac transported cumulatively across the cornea from cubosomal formulation and Ketorolac solution, respectively.

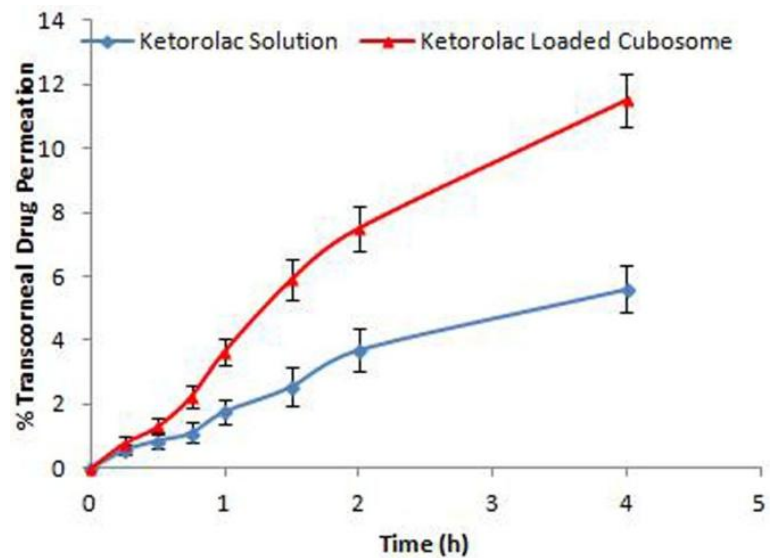

Fig. 4: Cumulative transcorneal permeation of Ketorolac loaded cubosome through the excised goat cornea.

\section{In vitro corneal retention study}

Ketorolac concentration was estimated into the cornea prior treated with ketorolac solution and ketorolac loaded cubosomes. It was observed that cornea treated with ketorolac loaded cubosomes $(0.291 \mu \mathrm{g} / \mathrm{mg})$ revealed significantly high drug content into the cornea when compared with ketorolac solution $(0.13 \mu \mathrm{g} / \mathrm{mg}), \mathrm{p}<0.01$. Significantly high drug concentration into cornea treated with ketorolac loaded cubosomes probably due to mucoadhesiveness of ketorolac loaded cubosomes followed by higher penetration and prolonged retention inside the cornea (Zaki et al., 2011).

\section{Ocular tolerance studies (Histopathology)}

On performing histological examination of goat cornea, it revealed the existence of normal ocular structures (Fig. 5) in the negative control $(0.9 \%, w / \mathrm{v} \mathrm{NaCl})$ and the drug containing cubosomal formulations. There were no any changes observed in the epidermal layer after internalization of cubosomal formulation inside cornea (Fig. 5C). Positive control (1\%, w/v SLS), treated corneal sample showed destruction of epithelial cells (Fig. 5B). On the basis of these observations it was concluded that the optimized formulation (ketorolac loaded cubosomes) was safe for ocular administration.

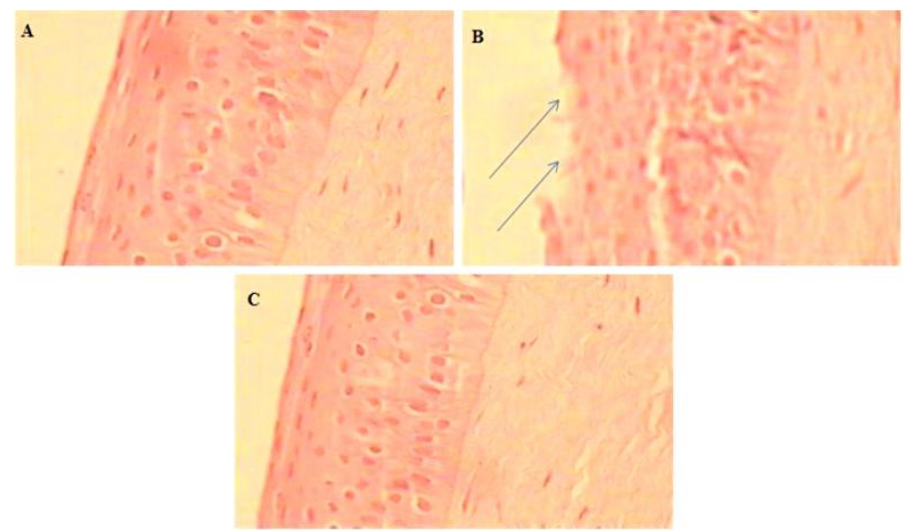

Fig. 5: Light microscopy of (A) Negative control $(0.9 \% \mathrm{NaCl})$, (B) Positive control $(1 \% \mathrm{w} / \mathrm{v}, \mathrm{SLS})$ and (C) Ketorolac loaded cubosome treated cross sections of excised goat corneal tissue. 


\section{CONCLUSION}

Ketorolac loaded cubosomes were successfully developed and optimized by using glyceryl mono-oleate and poloxamer 407. Optimized formulation expressed particles size in desired nano size range with good encapsulation of ketorolac. In addition, significantly high transcorneal permeation and retention of ketorolac was achieved by the developed cubosomal formulation. Histopathology study revealed that ketorolac loaded cubosomes was safe for ocular use. It can be concluded that the optimized formulation could be an excellent substitute to conventional eye drops, however there is need of some more finding in terms of in vivo results to make it clinically viable.

\section{ACKNOWLEDGEMENT}

The authors are thankful to Prof. Farhan J Ahmad, Faculty of Pharmacy, Hamdard University, New Delhi for providing zetasizer and image processing facilities.

Financial support and sponsorship: Nil.

Conflict of Interests: There are no conflicts of interest.

\section{REFERENCES}

Abdullah T.A., Ibrahim N.J., Warsi M.H. Chondroitin sulfatechitosan nanoparticles for ocular delivery of bromfenac sodium: Improved permeation, retention, and penetration. Int J Pharm Invest 2016; 6: 96-105

Anitha A., Deepa N., Chennazhi K. P., Nair S.V., Tamura H., Jayakumar R. Development of mucoadhesive thiolated chitosan nanoparticles for biomedical applications. Carbohydr Polym 2011; 83: 6673.

Araújo J., Vega E., Lopes C., Egea M.A., Garcia M.L., Souto E.B. Effect of polymer viscosity on physicochemical properties and ocular tolerance of FB-loaded PLGA nanospheres. Colloids Surf B Biointerfaces 2009; 72: 48-56

Badawi A.A., El-Laithy H.M., El Qidra R.K., El Mofty H., El dally M. Chitosan based nanocarriers for indomethacin ocular delivery. Arch Pharm Res 2008; 31: 1040-9

Bourlais C.L., Acar L., Zia H., Sado P.A., Needham T., Leverge R. Ophthalmic drug delivery systems--recent advances. Prog Retin Eye Res 1998; 17: 33-58

Boyd B.J. Characterization of drug release from cubosomes using the pressure ultrafiltration method. Int J Pharm 2003; 260: 239-47

Chien Y.W. Ocular drug delivery and delivery systems. In: Novel drug delivery systems. 2 nd ed. New York: Marcel Dekker; 1992; 269-70

Chopra S., Patil G.V., Motwani S.K. Release modulating hydrophilic matrix systems of losartan potassium: optimization of formulation using statistical experimental design. Eur J Pharm Biopharm 2007; 66: 73-82

Davies N.M., Farr S.J., Hadgraft J., Kellaway I.W. Evaluation of mucoadhesive polymers in ocular drug delivery. II. Polymer-coated vesicles. Pharm Res 1992; 9(9): 1137-44
De Campos A.M., Diebold Y., Carvalho E.L., Sánchez A., Alonso M.J. Chitosan nanoparticles as new ocular drug delivery systems: In vitro stability, in vivo fate, and cellular toxicity. Pharm Res 2004; 21 : 803-10

Dodane V., Amin Khan M., Merwin J.R. Effect of chitosan on epithelial permeability and structure. Int J Pharm 1999; 182: 21-32

Encyclopedia of controlled drug delivery. 1999; 2: 605-622

Felt O., Baeyens V., Zignani M., Buri P., and Gurny R. Mucosal drug delivery- ocular-

Garg G., Saraf S., Saraf S. Cubosomes: an overview. Biol Pharm Bull 2007; 30: 350-3

Guinedi A.S., Mortada N.D., Mansour S., Hathout R.M. Preparation and evaluation of reverse-phase evaporation and multilamellar niosomes as ophthalmic carriers of acetazolamide. Int J Pharm 2005; 306: $71-82$

Gupta H., Aqil M., Khar R.K., Ali A., Bhatnagar A., Mittal G. Sparfloxacin-loaded PLGA nanoparticles for sustained ocular drug delivery. Nanomedicine 2010; 6: 324-33

Gustafsson J., Ljusberg-Wahren H., Almgren M., Larsson K. Cubic lipid-water phase dispersed into submicron particles. Langmuir 1996; 12: 4611-3

Jain G.K., Pathan S.K., Akhter S., Jayabalan N., Talegaonkar S., Khar R.K., Ahmad F.J. Colloids Surf. B Biointerfaces 82 (2011) 397 403

Larsson K. Aqueous dispersion of cubic lipid water phases. Curr Opin Colloid Interface Sci 2000; 5: 64-9

Magenheim B., Levy M.Y., Benita S. A new In vitro technique for the evaluation of drug release profile from colloidal carriersultrafiltration technique at low pressure. Int J Pharm 1993; 94: 115-23

Malmsten M. Soft drug delivery systems. Soft Matter 2006; 2: $760-9$

Tsukada K., Ueda S., Okada R. Preparation of liposomeencapsulated anti-tumor drugs; relationship between lipophilicity of drugs and In vitro drug release. Chem Pharma Bull 1984; 32: 19: 29-35

Warsi M. H., Anwar M., Garg V., Jain G. K., Talegaonkar S., Ahmad F. J., Khar R. K. Dorzolamide-loaded PLGA/vitamin E TPGS nanoparticles for glaucoma therapy: Pharmacoscintigraphy study and evaluation of extended ocular hypotensive effect in rabbits. Colloids Surf B Biointerfaces. 2014; 122: 423-431

Wörle G., Drechsler M., Koch M.H., Siekmann B., Westesen K., Bunjes H. Influence of composition and preparation parameters on the properties of aqueous monoolein dispersions. Int J Pharm 2007; 329: 1507

Zaki Randa., Khaled M. Hosny., Ahmed Khames., Ahmed Abdelbary. Ketorolac Tromethamine In-situ Ocular Hydro Gel; Preparation, Characterization, and In-vivo Evaluation. International Journal of Drug Delivery. 2011; 3: 535-545

Zhao X.Y., Zhang J., Zheng L.Q., Li DH. Studies of cubosomes as a sustained drug delivery system. J Disp Sci Tech 2004; 25: 795-9

How to cite this article:

Ali Z, Sharma PK, Warsi MH. Fabrication and Evaluation of Ketorolac Loaded Cubosome for Ocular Drug Delivery. J App Pharm Sci, 2016; 6 (09): 204-208. 